\title{
Signatures of complex new physics in charged current $B$ anomalies
}

\author{
Suman Kumbhakar* \\ Centre for High Energy Physics, Indian Institute of Science, Bangalore 560012, India \\ E-mail: ksumandisc.ac.in
}

Present world averages of $R_{D}$ and $R_{D^{*}}$ measurements are in disagreement with their Standard Model (SM) predictions at a level of $\sim 3.1 \sigma$. Assuming the new physics Wilson coefficients to be complex, we do a global fit to the present $b \rightarrow c \tau \bar{v}$ data. We find that there are only one/two/three allowed solutions depending upon three choices on upper limits 10\%/30\%/60\% of $\operatorname{Br}\left(B_{c} \rightarrow \tau \bar{v}\right)$. Further we calculate the maximum values of $\mathrm{CP}$ violating triple product asymmetries in $B \rightarrow$ $D^{*} \tau \bar{v}$ decay allowed by the current data. We find that one of the three asymmetries can be enhanced up to only $\sim 2-3 \%$ due to presence of the allowed new physics solutions.

40th International Conference on High Energy physics - ICHEP2020

July 28 - August 6, 2020

Prague, Czech Republic (virtual meeting)

${ }^{*}$ Speaker. 


\section{Introduction}

The semileptonic $B$ meson decays are a very fertile candidates to probe possible physics beyond the Standard Model (SM). In past few years, several measurements by BaBar, Belle and LHCb in the charged current $b \rightarrow c \ell \bar{v}$ transitions show significant deviations from their SM predictions. In this sector, the main discrepancies are observed in the following two flavor ratios

$$
R_{D}=\frac{\mathscr{B}(B \rightarrow D \tau \bar{v})}{\mathscr{B}(B \rightarrow D\{e / \mu\} \bar{v})}, \quad R_{D^{*}}=\frac{\mathscr{B}\left(B \rightarrow D^{*} \tau \bar{v}\right)}{\mathscr{B}\left(B \rightarrow D^{*}\{e / \mu\} \bar{v}\right)}
$$

The present world averages of $R_{D}-R_{D^{*}}$ are about $\sim 3.1 \sigma$ higher than their SM predictions [1]. This discrepancy is an indication of lepton flavor universality (LFU) violation between $\tau$ and $\mu / e$ leptons. This is further corroborated in the measurement of another flavor ratio $R_{J / \psi}=\Gamma\left(B_{c} \rightarrow\right.$ $J / \psi \tau \bar{v}) / \Gamma\left(B_{c} \rightarrow J / \psi \mu \bar{v}\right)$ by LHCb collaboration which is found to be $\sim 1.7 \sigma$ higher than its SM value [2]. These deviations could be due to presence of new physics (NP) either in $b \rightarrow c \tau \bar{v}$ or in $b \rightarrow c\{\mu, e\} \bar{v}$ transition. However, it has been shown in Ref. [3] that the latter possibility is ruled out by other measurements. Therefore, we assume the presence of NP only in $b \rightarrow c \tau \bar{v}$ transition. Apart from these, the Belle collaboration has measured two angular observables in the $B \rightarrow D^{*} \tau \bar{v}$ decay - (a) the $\tau$ polarization $P_{\tau}^{D^{*}}$ and (b) the $D^{*}$ longitudinal polarization fraction $F_{L}^{D^{*}}$. The measured value of $P_{\tau}^{D^{*}}$ is consistent with its SM prediction [4] whereas for $F_{L}^{D^{*}}$ it is $1.6 \sigma$ higher than its $\mathrm{SM}$ value [5].

Recently, the anomalies in $b \rightarrow c \tau \bar{v}$ transition have been studied in various model independent techniques, see for example Ref. [6] and the references therein. In the most of these analysis, the NP Wilson coefficients (WCs) are assumed to be real. These NP WCs are determined by doing a fit to the data available in this sector along with the constraint on the branching ratio of $B_{c} \rightarrow \tau \bar{v}$ decay. In Ref. [6], it has been shown that the NP Lorentz structure in form of $(V-A) \times(V-A)$ is the only one operator solution allowed by the present data.

In this paper we do a global fit to all present data on $b \rightarrow c \tau \bar{v}$ transition by starting with a most general effective Hamiltonian. Assuming the NP WCs to be complex, we find the allowed NP solutions with their corresponding WCs. We show that one/two/three NP solution(s) is (are) allowed if we consider three different upper limits $10 \% / 30 \% / 60 \%$ on the branching ratio of $B_{c} \rightarrow$ $\tau \bar{v}$. Further, we compute the predictions of the CP violating triple product asymmetries in $B \rightarrow$ $D^{*} \tau \bar{v}$ decay for the three NP solutions. We show that one of these three asymmetries can be enhanced up to $\sim 2-3 \%$ in presence the allowed NP scenarios. The talk, given in the conference, is based on Ref. [7].

The paper is organized as follows. In Section II, we describe our methodology for calculation and present our fit results. In section III, we determine the maximum possible CP violating triple product asymmetries in $B \rightarrow D^{*} \tau \bar{v}$ decay allowed by the current data. We present our conclusions in section IV.

\section{New physics solutions}

We start with the most general effective Hamiltonian for $b \rightarrow c \tau \bar{v}$ transition which contains 
all possible Lorentz structures. This is expressed as [3]

$$
\mathscr{H}_{\mathrm{eff}}=\frac{4 G_{F}}{\sqrt{2}} V_{c b}\left[\mathscr{O}_{V_{L}}+\frac{\sqrt{2}}{4 G_{F} V_{c b}} \frac{1}{\Lambda^{2}}\left\{\sum_{i}\left(C_{i} \mathscr{O}_{i}+C_{i}^{\prime} \mathscr{O}_{i}^{\prime}+C_{i}^{\prime \prime} \mathscr{O}_{i}^{\prime \prime}\right)\right\}\right],
$$

where $G_{F}$ is the Fermi coupling constant and $V_{c b}$ is the Cabibbo-Kobayashi-Maskawa (CKM) matrix element. Here we assume that the neutrino is left chiral. We also assume the new physics scale $\Lambda=1 \mathrm{TeV}$. The Lorentz structures of these three sets of operators are described in Ref. [3]. In particular, $\mathscr{O}_{i}^{\prime}$ and $\mathscr{O}_{i}^{\prime \prime}$ operators can be expressed in terms of five unprimed operators using Fierz identity. The constants $C_{i}, C_{i}^{\prime}$ and $C_{i}^{\prime \prime}$ are the respective WCs of the NP operators in which NP effects are hidden. In this analysis, we assume these NP WCs to be complex.

Using the effective Hamiltonian given in Eq. (2.1), we calculate the expressions of measured observables $R_{D}, R_{D^{*}}, R_{J / \psi}, P_{\tau}^{D^{*}}$ and $F_{L}^{D^{*}}$ as functions of the NP WCs. To obtain the values of NP WCs, we do a fit of these expressions to the measured values of the observables. In doing the fit, we take only one NP operator at a time. We define the $\chi^{2}$ function as follows

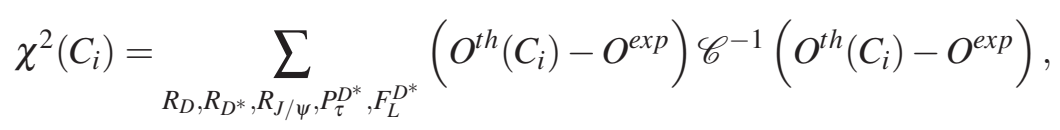

where $O^{\text {th }}\left(C_{i}\right)$ are NP predictions of each observable and $O^{\exp }$ are the corresponding experimental central values. The $\mathscr{C}$ denotes the covariance matrix which includes both theory and experimental correlations.

To obtain the values of NP WCs, we minimize the $\chi^{2}$ function by taking non-zero value of one NP WC at a time. While doing so, we set other coefficients to be zero. This minimizations is performed by the CERN MINUIT library [8]. We allow only those NP WCs which satisfy $\chi_{\min }^{2} \leq 4.5$. The central values of these allowed WCs of NP solutions are listed in Table 1. Further the purely

\begin{tabular}{|c|c|c|c|}
\hline \hline NP type & Best fit value(s) & $\chi_{\min }^{2}$ & pull \\
\hline$C_{V_{L}}$ & $0.10 \pm 0.12 i$ & 4.55 & 4.1 \\
\hline$C_{S_{L}}^{\prime}$ & $0.25 \pm 0.86 i$ & 4.50 & 4.2 \\
\hline$C_{T}^{\prime \prime}$ & $0.06 \pm 0.09 i$ & 3.45 & 4.3 \\
\hline$C_{S_{L}}$ & $-0.82 \pm 0.45 i$ & 2.50 & 4.4 \\
\hline \hline
\end{tabular}

Table 1: Best fit values of NP WCs at $\Lambda=1 \mathrm{TeV}$ which can account for present $b \rightarrow c \tau \bar{v}$ data. We have $\chi_{\mathrm{SM}}^{2}=21.80$ and pull $=\sqrt{\chi_{\mathrm{SM}}^{2}-\chi_{\min }^{2}}$.

leptonic decay $B_{c} \rightarrow \tau \bar{v}$ plays a crucial role to constrain the NP solutions, in particular for the pseudo-scalar operators. Therefore, these NP operators are highly constrained by this observable. The SM predicts this branching fraction to be $\sim 2.15 \times 10^{-2}$.

In Ref. [9], the upper limit on this branching ratio is set to be $10 \%$ from the LEP data which are admixture of $B_{c} \rightarrow \tau \bar{v}$ and $B_{u} \rightarrow \tau \bar{v}$ decays at $Z$ peak. On the other hand, the authors of Ref. [10] obtained this upper limit to be $30 \%$ by making use of the lifetime of $B_{c}$ meson. This is estimated by considering that the $B_{c} \rightarrow \tau \bar{v}$ decay rate does not exceed the fraction of the total width which is allowed by the calculation of the lifetime in the SM. In Ref. [11], the authors have argued that these two different upper limits are too conservative and these could be over-estimated. However, 
taking all uncertainties into account the decay width of $B_{c}$ meson can be relaxed up to $60 \%$ which is not that much conservative. Therefore, we consider these three different upper limits on branching ratio of $B_{c} \rightarrow \tau \bar{v}$ to constrain the NP parameter space.
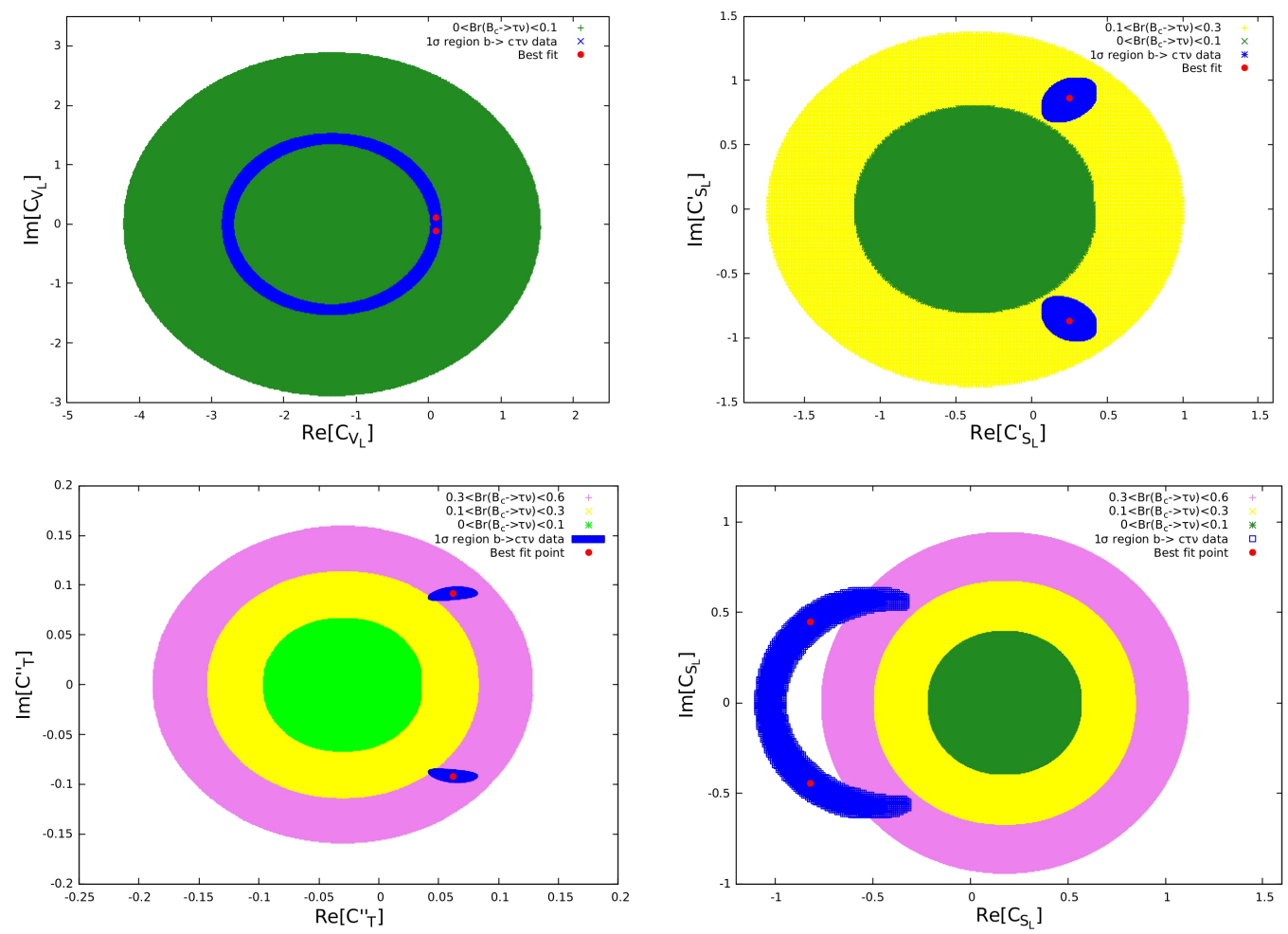

Figure 1: The $1 \sigma$ regions allowed by $b \rightarrow c \tau \bar{v}$ data (blue) and parameter spaces for three different upper limits $10 \%$ (green), $30 \%$ (yellow), 60\% (violet) of $B r\left(B_{c} \rightarrow \tau \bar{v}\right)$ for each complex NP WC listed in Table 1. In each plot, the red dots represent the best fit points.

In Fig. 1, we have shown the parameter space which span $1 \sigma$ region allowed by present $b \rightarrow$ $c \tau \bar{v}$ data, three different ranges of branching ratio of $B_{c} \rightarrow \tau \bar{v}$ and the best fit point for each solution listed in Table 1. Only the $\mathscr{O}_{V_{L}}$ solution falls within the allowed space constrained by $\operatorname{Br}\left(B_{c} \rightarrow \tau \bar{v}\right)<10 \%$. The allowed $1 \sigma$ regions for $\mathscr{O}_{S_{L}}^{\prime}$ and $\mathscr{O}_{T}^{\prime \prime}$ solutions fall into the regions allowed by the constraints $\operatorname{Br}\left(B_{c} \rightarrow \tau \bar{v}\right)<30 \%$ and $<60 \%$ respectively. The best fit NP WCs of $\mathscr{O}_{S_{L}}$ solution do not fall into the region allowed by the constraint $\operatorname{Br}\left(B_{c} \rightarrow \tau \bar{v}\right)<60 \%$ whereas a small fraction of the $1 \sigma$ region overlaps with the region allowed by $30 \%<\operatorname{Br}\left(B_{c} \rightarrow \tau \bar{v}\right)<60 \%$. Hence the $\mathscr{O}_{S_{L}}$ solution is mildly disfavored.

\section{CP violating triple product asymmetries}

In this section, we focus on CP violating triple product asymmetries (TPA) in $B \rightarrow D^{*} \tau \bar{v}$ decay. The full angular distribution of quasi-four body decay $B \rightarrow D^{*}(\rightarrow D \pi) \tau \bar{v}$ can be described by four independent parameters - (a) $q^{2}=\left(p_{B}-p_{D^{*}}\right)^{2}$ where $p_{B}$ and $p_{D^{*}}$ are respective four momenta of $B$ and $D^{*}$ meson, (b) $\theta_{D}$ the angle between $B$ and $D$ mesons where $D$ meson comes from $D^{*}$ decay, (c) $\theta_{\tau}$ the angle between $\tau$ momenta and $B$ meson, and (d) $\phi$ the angle between $D^{*}$ decay plane 
and the plane defined by the $\tau$ momenta. The triple products (TP) are obtained by integrating the full decay distribution in different ranges of the polar angles $\theta_{D}$ and $\theta_{\tau}[12]$. These TPs $A_{T}^{(i)}$ are the angular coefficients of $\sin \phi$ and $\sin 2 \phi$ and these are odd under the CP transformation which leads to these quantities to be $\mathrm{CP}$ violating observables. These three TPs are defined as follows [12]:

$$
A_{T}^{(1)}\left(q^{2}\right)=\frac{4 V_{5}^{T}}{A_{L}+A_{T}}, \quad A_{T}^{(2)}\left(q^{2}\right)=\frac{V_{3}^{0 T}}{A_{L}+A_{T}}, \quad A_{T}^{(3)}\left(q^{2}\right)=\frac{V_{4}^{0 T}}{A_{L}+A_{T}}
$$

where $V$ 's are the angular coefficients and $A_{L}$ and $A_{T}$ are the longitudinal and transverse amplitudes respectively, defined in Ref. [13]. The SM predictions of these TP are almost zero. Therefore, the complex NP WCs can predict a non-zero value for these quantities. For the CP conjugate decay, the definitions in Eq. (3.1) take the following forms

$$
\bar{A}_{T}^{(1)}\left(q^{2}\right)=-\frac{4 \bar{V}_{5}^{T}}{\bar{A}_{L}+\bar{A}_{T}}, \quad \bar{A}_{T}^{(2)}\left(q^{2}\right)=\frac{\bar{V}_{3}^{0 T}}{\bar{A}_{L}+\bar{A}_{T}}, \quad \bar{A}_{T}^{(3)}\left(q^{2}\right)=-\frac{\bar{V}_{4}^{0 T}}{\bar{A}_{L}+\bar{A}_{T}} .
$$

Using Eqs. (3.1) and (3.2), three asymmetries can be defined between the corresponding TPs of the decay and its CP conjugate. These TPAs are defined as follows

$$
\begin{aligned}
\left\langle A_{T}^{(1)}\left(q^{2}\right)\right\rangle & =\frac{1}{2}\left(A_{T}^{(1)}\left(q^{2}\right)+\bar{A}_{T}^{(1)}\left(q^{2}\right)\right), \\
\left\langle A_{T}^{(2)}\left(q^{2}\right)\right\rangle & =\frac{1}{2}\left(A_{T}^{(2)}\left(q^{2}\right)-\bar{A}_{T}^{(2)}\left(q^{2}\right)\right), \\
\left\langle A_{T}^{(3)}\left(q^{2}\right)\right\rangle & =\frac{1}{2}\left(A_{T}^{(3)}\left(q^{2}\right)+\bar{A}_{T}^{(3)}\left(q^{2}\right)\right) .
\end{aligned}
$$
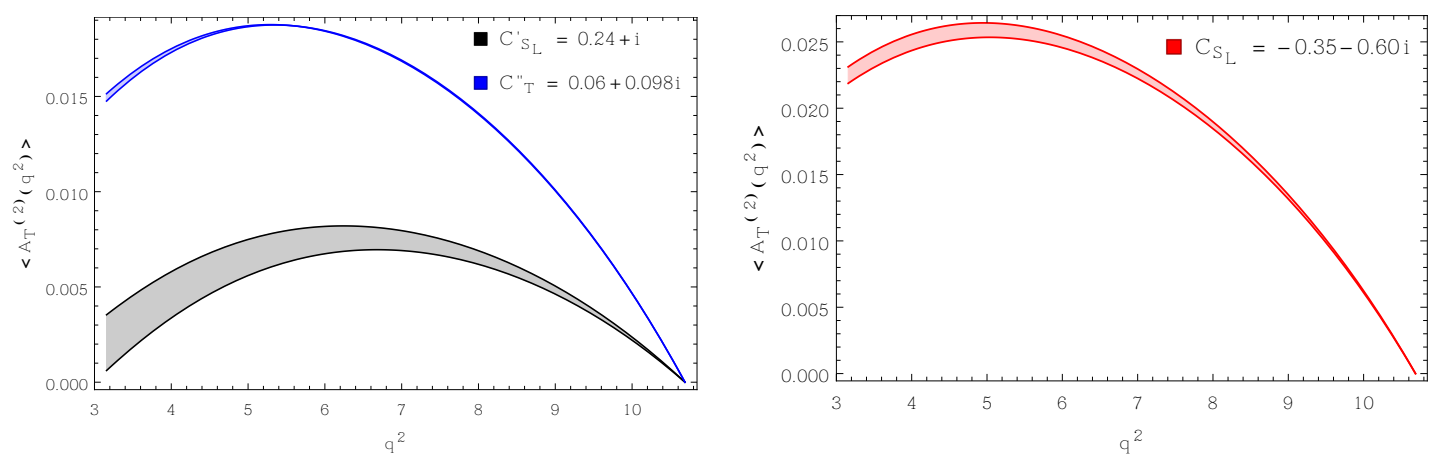

Figure 2: The second TPA is plotted as a function of $q^{2}\left(\mathrm{GeV}^{2}\right)$ for three benchmark NP WCs $C_{S_{L}}^{\prime}=0.24+i$ (blue curve), $C_{T}^{\prime \prime}=0.06+0.098 i$ (black curve) and $C_{S_{L}}=-0.35-0.60 i$ (red curve).

Our next aim is to compute the maximum CP violation allowed by the present $b \rightarrow c \tau \bar{v}$ data. To calculate this, we choose a benchmark point from the $1 \sigma$ allowed parameter space of each NP solution. The TPAs $\left\langle A_{T}^{(1)}\left(q^{2}\right)\right\rangle$ and $\left\langle A_{T}^{(3)}\left(q^{2}\right)\right\rangle$ depend only on the $\mathscr{O}_{V_{L}}$ operator and it has the same Lorentz structure as the SM. Therefore, the $\mathscr{O}_{V_{L}}$ solution predicts these two asymmetries to be zero for whole $q^{2}$ range. For other two allowed NP solutions, the predictions are zero because these two asymmetries do not depend on those NP WCs. Only the second TPA $\left\langle A_{T}^{(2)}\left(q^{2}\right)\right\rangle$ is non-zero for the $\mathscr{O}_{S_{L}}^{\prime}$ and $\mathscr{O}_{T}^{\prime \prime}$ solutions. Therefore, we pick a benchmark points from Fig 1 for each of these two solutions. These points are $C_{S_{L}}^{\prime}=0.24 \pm i$ and $C_{T}^{\prime \prime}=0.06 \pm 0.098 i$, which can lead to the 
maximum value of the TPA $\left\langle A_{T}^{(2)}\left(q^{2}\right)\right\rangle$ in $B \rightarrow D^{*} \tau \bar{v}$ decay. In the left panel of Fig. 2, we plot the TPA $\left\langle A_{T}^{(2)}\left(q^{2}\right)\right\rangle$ as a function of $q^{2}$ for these two benchmark points of $\mathscr{O}_{S_{L}}^{\prime}$ and $\mathscr{O}_{T}^{\prime \prime}$ solutions. From this plot, we observe that $\left\langle A_{T}^{(2)}\left(q^{2}\right)\right\rangle$ reaches a maximum value of $\sim 0.7 \%$ at $q^{2} \simeq 6 \mathrm{GeV}^{2}$ for the $\mathscr{O}_{S_{L}}^{\prime}$ solution whereas it reaches a maximum value of $\sim 1.7 \%$ at $q^{2} \simeq 5.4 \mathrm{GeV}^{2}$ for the $\mathscr{O}_{T}^{\prime \prime}$ solution.

As per discussion in Sec II, a small fraction of the $1 \sigma$ region of the marginally disfavored $\mathscr{O}_{S_{L}}$ solution falls on the region spanned by the constraint $30 \%<\operatorname{Br}\left(B_{c} \rightarrow \tau \bar{v}\right)<60 \%$. For completeness, we calculate the predictions of TPAs for this solution. We can get a allowed value of $C_{S_{L}}$ which can give to maximum possible TPA for the $\left\langle A_{T}^{(2)}\left(q^{2}\right)\right\rangle$. We choose a benchmark point $C_{S_{L}}=-0.35-0.60 i$ from the allowed region and calculate the second TPA. In right panel of Fig. 2, we plot $\left\langle A_{T}^{(2)}\left(q^{2}\right)\right\rangle$ as a function of $q^{2}$ for the benchmark point of $C_{S_{L}}$. From this plot, we observe that the second TPA reaches a maximum value of $\sim 2.6 \%$ at $q^{2} \simeq 5 \mathrm{GeV}^{2}$ and decreases to zero at $q_{\max }^{2}$. In fact, this is the maximum value of $\left\langle A_{T}^{(2)}\left(q^{2}\right)\right\rangle$ predicted by the scalar operator solution among all the predictions made by allowed NP solutions.

\section{Summary}

Assuming the NP WCs to be complex, we have done a global fit of present $b \rightarrow c \tau \bar{v}$ data. We find that the $\mathscr{O}_{V_{L}}$ solution is the only NP solution allowed by the constraint $\operatorname{Br}\left(B_{c} \rightarrow \tau \bar{v}\right)<10 \%$. If we relax the constraint to $30 \%$ or $60 \%$, then we get one or two additional allowed NP solutions. We then compute the maximum values of CP violating TPAs in $B \rightarrow D^{*} \tau \bar{v}$ decay for the allowed NP solutions. We find that the predictions of first and third TPAs are zero for all NP solutions whereas the second TPA reaches a maximum value of $\sim 1.9 \%$ for the $\mathscr{O}_{S_{L}}^{\prime}$ solution and $\sim 0.9 \%$ for the $\mathscr{O}_{T}^{\prime \prime}$ solution. The mildly favored NP solution $\mathscr{O}_{S_{L}}$ predicts a maximum value of $\sim 2.6 \%$ for the second TPA which is the maximum predicted value among all the NP predictions.

\section{References}

[1] Y. S. Amhis et al. [HFLAV], [arXiv:1909.12524 [hep-ex]].

[2] R. Aaij et al. [LHCb], Phys. Rev. Lett. 120 (2018) no.12, 121801

[3] A. K. Alok, D. Kumar, J. Kumar, S. Kumbhakar and S. U. Sankar, JHEP 09 (2018), 152

[4] S. Hirose et al. [Belle], Phys. Rev. Lett. 118 (2017) no.21, 211801

[5] A. Abdesselam et al. [Belle], [arXiv:1903.03102 [hep-ex]].

[6] A. K. Alok, D. Kumar, S. Kumbhakar and S. Uma Sankar, Nucl. Phys. B 953 (2020), 114957

[7] S. Kumbhakar, [arXiv:2007.08132 [hep-ph]].

[8] F. James and M. Roos, Comput. Phys. Commun. 10 (1975), 343-367

[9] A. G. Akeroyd and C. H. Chen, Phys. Rev. D 96 (2017) no.7, 075011

[10] R. Alonso, B. Grinstein and J. Martin Camalich, Phys. Rev. Lett. 118 (2017) no.8, 081802

[11] M. Blanke, A. Crivellin et al., Phys. Rev. D 99 (2019) no.7, 075006

[12] M. Duraisamy and A. Datta, JHEP 09 (2013), 059

[13] M. Duraisamy, P. Sharma and A. Datta, Phys. Rev. D 90 (2014) no.7, 074013 